\section{Small cell lung cancer mimicking a pulmonary venous angiosarcoma}

A 67-year-old man presented with dyspnoea and non-specific chest pain. A CT scan showed a mass lesion within the right superior pulmonary vein protruding into the left atrium (fig $1 \mathrm{~A}$, B). Positron emission tomography with ${ }^{18}$ fluoro-2-deoxy-Dglucose showed uptake within the lesion but no extrathoracic disease (fig 1C). A clinical-radiological diagnosis of pulmonary venous angiosarcoma was made and surgical resection was offered for symptom palliation. At surgery the tumour mass was found to be growing from the right superior pulmonary vein into the left atrium (fig 2A). No lung mass was seen intraoperatively. A right pneumonectomy with en bloc resection of the left atrium and posterior wall of the right atrium was performed in order to achieve clear resection margins. Unexpectedly, histological examination showed small cell lung cancer and microscopic examination revealed a tiny focus of tumour within the adjacent lung parenchyma. The tumour had grown into the pulmonary vein and then extended along it into the left atrium (fig 2B). The pathological staging was pT4 pN0 $\mathrm{pMx}$. The patient recovered well from surgery and received four cycles of adjuvant chemotherapy postoperatively.

\section{Learning points}

- Lung cancer can invade and grow within the pulmonary veins mimicking angiosarcoma.

- When possible, a preoperative or intraoperative biopsy should be considered before embarking on a pneumonectomy.

- Surgical resection using cardiopulmonary bypass should be considered for palliation of symptoms.

Invasion of the atria by carcinoma of the lung is uncommon and usually occurs by direct invasion. Although there are reports of lung cancer growing into the left atrium via the pulmonary veins, there is usually an obvious parenchymal lung mass. ${ }^{1}$ This case is unusual in that the tumour had arisen from a small focus within the lung parenchyma adjacent to the vein and then grown within the vein, mimicking an angiosarcoma. Surgery for angiosarcoma can produce an acceptable survival rate with palliation of symptoms. ${ }^{2}$ However, had a preoperative diagnosis of small cell lung cancer been possible, this patient would have been offered primary chemotherapy. He did, however, achieve excellent palliation of symptoms with surgery.
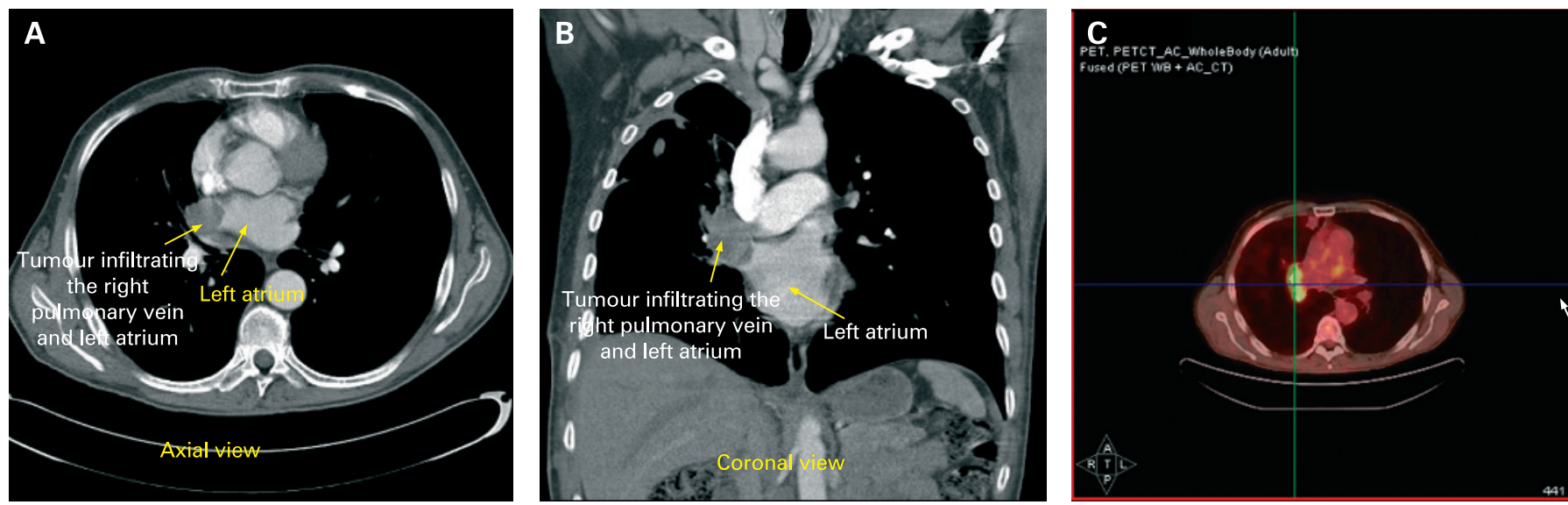

Figure 1 ( $A, B) C T$ scan showing the tumour infiltrating the right superior pulmonary vein and left atrium. (C) Positron emission tomography showing uptake in the right superior pulmonary vein and left atrium.

Figure 2 (A) Macroscopic view of the excised tumour. (B) H\&E stained slide of small cell lung cancer in the pulmonary vein originating from the lung parenchyma.
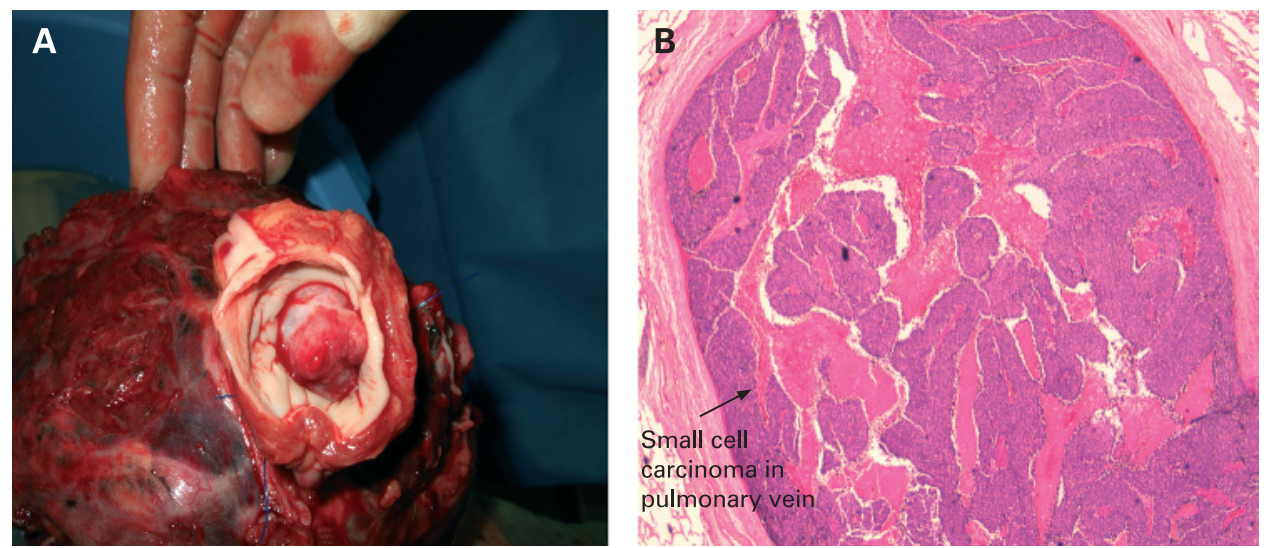


\section{J B Morjaria, ${ }^{1}$ C K Choong, ${ }^{2}$ K Amsha, ${ }^{3}$ S Stewart, ${ }^{4}$ F C Wells, ${ }^{2}$ R C Rintoul ${ }^{1}$}

${ }^{1}$ Department of Thoracic Oncology, Papworth Hospital, Cambridge, UK; ${ }^{2}$ Department of Cardiothoracic Surgery, Papworth Hospital, Cambridge, UK; ${ }^{3}$ Department of CardioRespiratory Medicine, King's Mill Hospital, Nottinghamshire, UK; ${ }^{4}$ Department of Pathology, Papworth Hospital, Cambridge, UK

Correspondence to: Dr R C Rintoul, Department of Thoracic Oncology, Papworth Hospital, Cambridge CB23 3RE, UK; robert.rintoul@papworth.nhs.uk

Competing interests: None.
Patient consent: Obtained.

Accepted 31 December 2008

Thorax 2009;64:827-828. doi:10.1136/thx.2008.109306

\section{REFERENCES}

1. Lestuzzi C, Viel E, Mimo R, et al. Left atrial invasion by lung carcinoma through a pulmonary vein. Int J Cardiovasc Imaging 2001;17:107-10.

2. Bacha EA, Wright $\mathrm{CD}$, Grillo $\mathrm{HC}$, et al. Surgical treatment of primary pulmonary sarcomas. Eur J Cardiothorac Surg 1999;15:456-60.

\section{Lung alert}

\section{Influenza A resistance to oseltamivir}

Oseltamivir is a neuraminidase inhibitor used for the treatment and prophylaxis of influenza A infections. This study investigated the differences in demographics, epidemiology, clinical symptoms, severity of illness and clinical outcomes among patients infected with oseltamivirresistant or oseltamivir-susceptible influenza A (H1N1) virus in the USA. Information about viral isolates and their subtypes, particularly H1N1, was collected using data submitted to the Centres for Disease Control and Prevention by US public health laboratories. Data about illnesses from both groups were collected over the telephone using a standardised questionnaire.

142 out of 1155 cases of influenza A (H1N1) tested were resistant to oseltamivir. Data were collected from 99 oseltamivir-resistant patients who agreed to participate and 182 oseltamivirsusceptible cases. Among resistant patients the median age was 19 years; five patients (5\%) were hospitalised and four patients (4\%) died. The comparison of the two groups showed a similar prevalence of underlying medical conditions, age distribution and clinical symptoms, and it showed that circulating resistant viruses were unrelated to oseltamivir use.

The US nationally adjusted cases of oseltamivir-resistant influenza A (H1N1) in the 2007-8 season were estimated to be $2 \%$; however, resistance increased in the early 2008-9 season, reaching $98.5 \%$ in 268 tested cases.

This study was limited by the small numbers of influenza A (H1N1) cases and differing methodologies for data collection. However, it highlights an important issue of the emergence of treatment-resistant influenza strains and the need for further antiviral development.

- Dharan NJ, Gubareva LV, Meyer JJ, et al for the Oseltamivir-Resistance Working Group. Infections with oseltamivir-resistant influenza A (H1N1) virus in the United States. JAMA 2009;301:1034-41.

\section{Ismail}

Correspondence to: Dr I Ismail, CT2, University Hospital of South Manchester NHS Foundation Trust, Manchester, UK; iyad75@hotmail.co.uk 